OPEN ACCESS

Edited by:

Agata Gadaleta,

Università degli Studi di Bari Aldo

Moro, Italy

Reviewed by:

Zhanguo Xin

Agricultural Research Service (USDA),

United States

Arkadiusz Kosmala,

Institute of Plant Genetics (PAN),

Poland

*Correspondence:

Gábor Galiba

galiba.gabor@agrar.mta.hu

Specialty section: This article was submitted to

Plant Breeding,

a section of the journal

Frontiers in Plant Science

Received: 21 September 2017

Accepted: 14 November 2017

Published: 29 November 2017

Citation:

Kalapos B, Novák A, Dobrev P, Vitámvás $P$, Marincs F, Galiba $G$ and Vanková $R$ (2017) Effect of the Winter

Wheat Cheyenne 5A Substituted

Chromosome on Dynamics

of Abscisic Acid and Cytokinins in Freezing-Sensitive Chinese Spring

Genetic Background.

Front. Plant Sci. 8:2033.

doi: 10.3389/fpls.2017.02033

\section{Effect of the Winter Wheat Cheyenne 5A Substituted Chromosome on Dynamics of Abscisic Acid and Cytokinins in Freezing-Sensitive Chinese Spring Genetic Background}

\author{
Balázs Kalapos ${ }^{1,2}$, Aliz Novák1,2, Petre Dobrev³, Pavel Vítámvás ${ }^{4}$, Ferenc Marincs ${ }^{1,5}$, \\ Gábor Galiba ${ }^{1,2 *}$ and Radomira Vanková3
}

${ }^{1}$ Agricultural Institute, Centre for Agricultural Research, Hungarian Academy of Sciences, Martonvásár, Hungary, ${ }^{2}$ Festetics Doctoral School, Georgikon Faculty, University of Pannonia, Keszthely, Hungary, ${ }^{3}$ Institute of Experimental Botany, Academy of Sciences of the Czech Republic, Prague, Czechia, ${ }^{4}$ Department of Genetics and Plant Breeding, Crop Research Institute, Prague, Czechia, ${ }^{5}$ Agricultural Biotechnology Institute, National Agricultural Research and Innovation Centre, Gödöllö, Hungary

The effect of short- and long-term cold treatment on the abscisic acid (ABA) and cytokinin (CK) metabolism, and their main biosynthesis- and signaling-related genes were investigated in freezing-sensitive and freezing-tolerant wheat genotypes. Varieties Cheyenne and Chinese Spring substituted with the 5A Cheyenne chromosome, which represented freezing-tolerant genotypes, were compared with the freezing-sensitive Chinese Spring. Hormone levels and gene expression data indicated that the short- and long-term cold treatments are associated with specific regulation of the accumulation of cold-protective proteins and phytohormone levels, as well as the expression profiles of the hormone-related genes. The significant differences were observed between the genotypes, and between their leaf and crown tissues, too. The level of dehydrins, including WCS120 protein, and expression of WCS120 gene were considerably higher in the freezing-tolerant genotypes after 21 days of cold treatment. Expression of Cor14b and CBF14, cold-responsive regulator genes, was increased by cold treatment in all genotypes, to higher extent in freezing-tolerant genotypes. Cluster analysis revealed that the tolerant genotypes had a similar response to cold treatment, regarding expression of the ABA and CK metabolic genes, as well as hormone levels in leaves. As far as hormone levels in crowns are concerned, however, the strongly freezing-tolerant Cheyenne variety clustered separately from the Chinese Spring and the substitution line, which were more similar to each other after both 1 and 21 days of cold treatment than to Cheyenne. Based on these results we concluded that the $5 \mathrm{~A}$ chromosome of wheat might have both a direct and an indirect impact on the phytohormone-dependent cold-induced freezing tolerance. Based on the gene expression data, novel genetic markers could be developed, which may be used to determine the freezing tolerance level in a wide range of wheat varieties.

Keywords: cold treatment, freezing tolerance, abscisic acid, cytokinin, phytohormones, gene expression, Triticum aestivum 


\section{INTRODUCTION}

One of the major cereals at present is the hexaploid (AABBDD genomes) Triticum aestivum or common wheat (Dubcovsky and Dvorak, 2007), which combines the D genome from Aegilops tauschii with the $\mathrm{AB}$ genomes from tetraploid wheat and exhibits a broader adaptability to different environmental conditions in comparison with its predecessors. As T. aestivum is allohexaploid, containing $\mathrm{A}, \mathrm{B}$, and $\mathrm{D}$ genomes, all of its homeologous genes are triplicated. Consequently, common wheat tolerates the absence of one chromosome from any pair, without losing its fertility. This unique feature made it possible to develop a series of nullisomic lines from a moderate freezing-tolerant spring variety Chinese Spring (CS) (Sears, 1953). Using this nullisomic series, intervarietal chromosome substitution lines were created. In each of the substitution lines, one chromosome pair from the CS was replaced by the corresponding pair of chromosomes from the frost-resistant winter wheat variety Cheyenne $(\mathrm{Ch})$. Freezing test under natural and artificial conditions (Sutka, 1981; Sutka et al., 1986) confirmed earlier observations, which indicated that the chromosomes of 5th homologous group of Ch carry major genes controlling frost resistance (Cahalan and Law, 1979; Roberts, 1986). The relationship of cold hardening period and the level of frost resistance in different $\mathrm{CS} / \mathrm{Ch}$ chromosome substitution lines was also reported (Veisz and Sutka, 1989; Vágújfalvi et al., 1999). The biggest alteration between CS and CS(Ch5A) survival rate was registered after 19-day cold treatment, i.e., $19 \%$ in case of CS, $64 \%$ in case of CS $(\mathrm{Ch} 5 \mathrm{~A})$, while Ch had $100 \%$ survival (Vágújfalvi et al., 1999). Replacing chromosome 5A of CS with the corresponding chromosome from $\mathrm{Ch}[\mathrm{CS}(\mathrm{Ch} 5 \mathrm{~A})]$ increased the freezing tolerance of CS (Chen et al., 2009; Eagles et al., 2011).

A cluster of at least $12 \mathrm{C}$-repeat binding factor (CBF) genes, also known as dehydration responsive elements (DRE-binding factors), are in the frost resistance 2 locus (Fr-A2) on the long arm of chromosome 5A (Stockinger et al., 1997; Liu et al., 1998; Miller et al., 2006; Campoli et al., 2009), and therefore they were postulated to be responsible for freezing tolerance in wheat. The increased freezing tolerance of $\mathrm{CS}(\mathrm{Ch} 5 \mathrm{~A})$ was associated with increased transcription of the Ch CBF genes in the CS genetic background (Vágújfalvi et al., 2005). The target genes of $\mathrm{CBFs}$ include different transcription factors and effector genes belonging to the Cold-regulated (Cor) gene superfamily, the activation of which increases freezing tolerance (Pecchioni et al., 2014; Park et al., 2015). Among the genes of the CBF-cluster localized in the Fr-2 locus, CBF14 has a decisive influence on freezing tolerance, both in wheat and barley (Vágújfalvi et al., 2005; Fricano et al., 2009; Soltész et al., 2013; Dhillon and Stockinger, 2013). It is well established, that CBFs induce the expression of $H v C O R 14 b$ (Crosatti et al., 1996), and TaWCS120 (Vazquez-Tello et al., 1998), moreover they bind to the promoters of several other cold-regulated or droughtinducible genes in Triticeae (Cattivelli et al., 2002). These genes are differentially expressed in freezing-sensitive and freezingtolerant plants during cold acclimation (Sarhan et al., 1997; Crosatti et al., 2003; Vágújfalvi et al., 2003; Kosová et al., 2012).

The Cor $14 b$ gene encodes a polypeptide that is accumulated in the stroma fraction of the chloroplasts in the presence of light and its transcription was specifically induced by low temperature and light (Crosatti et al., 1999). The Cor $14 b$ was used as a marker gene to prove the effectiveness of cold acclimation in cereals (Knox et al., 2008; Dhillon et al., 2010; Gulyás et al., 2014). WCS120 belongs to the dehydrin (LEA II) gene family. The encoded WCS120 protein is supposed to be involved in low temperatureinduced physiological dehydration. The level of WCS120 was considered as a bio-marker of freezing tolerance in both coldacclimated (Vítámvás et al., 2007) and non-acclimated (Holková et al., 2009; Vítámvás et al., 2010; Kosová et al., 2013) winter wheats.

The first reaction of wheat plants to the sudden drop of temperature is the arrest of growth, followed by the adjustment of metabolism to the new, cold environment (acclimation phase). Phytohormones play a pivotal role in the coordination of this acclimation process (Galiba et al., 2013). One of the key players initiating the hormonal changes is the CBF-regulon itself, e.g., by enhancing the accumulation of the growth-repressing DELLA proteins (Achard et al., 2008).

In this paper we compare hormone and gene expression responses to 1 day cold-shock from previous work of our group (Kalapos et al., 2016) and the data obtained after 21 days of cold (this work) as a function of the chromosome 5A of Cheyenne in the CS genetic background. There are several reasons to choose these particular sampling points. First of all, when we compared the phytohormone responses of two common wheat cultivars (Kosová et al., 2012) and more recently of two einkorn lines (Vanková et al., 2014) during cold acclimation, characteristic changes specific for the individual stress response phases were found, which allowed optimizing the sampling times. The biggest alterations in the transcriptomes of CS and $\operatorname{CS}(\mathrm{Ch} 5 \mathrm{~A})$ substitution line were detected after 1 day cold treatment (Kocsy et al., 2010; Kalapos et al., 2016). As described above, the Ch5A related cold hardiness is almost fully manifested after 21-day cold treatment, with the lowest CS background effect. During this cold acclimation period, the plants still stay in the vegetative phase, which allowed us to avoid the hormonal changes associated with the developmental phase transition.

The environmental signals initiating the cold acclimation process are perceived by the leaves, but the survival of the plants depends on the fitness of meristems in the crowns (Hoffman et al., 2010). Due to the different functions of these organs, it is possible to expect different responses during cold acclimation. Indeed, when we recently analyzed the metabolic profiles, redox changes and gene expression levels in the crowns and leaves of CS and CS(Ch5A) substitution line, substantial differences were observed (Juhász et al., 2015; Boldizsár et al., 2016). These findings prompted us to study the phytohormone changes in both organs. Taking into account the intensive cross-talk among different phytohormones during cold acclimation, we focused on the interaction between the key hormone in abiotic stress responses - abscisic acid (ABA) and cytokinins (CKs), the hormones playing decisive role in plant stress acclimation. The evaluation of their metabolite contents has been complemented by determination of the expression pattern of selected hormone biosynthesis- and signaling-related genes. 


\section{MATERIALS AND METHODS}

\section{Plant Material and Treatments}

Seeds of Chinese Spring, Cheyenne and CS(Ch5A) substitution line (Veisz and Sutka, 1989; Vágújfalvi et al., 1999) were germinated for 5 days and then grown for 2 weeks under conditions described in Kalapos et al. (2016). The seedlings were grown in half-strength modified Hoagland solution under $16 \mathrm{~h}$ illumination at $270 \mu \mathrm{mol} \mathrm{m} \mathrm{m}^{-2} \mathrm{~s}^{-1}, 20 / 15^{\circ} \mathrm{C}$ day/night temperature and $70-75 \%$ relative humidity in a growth chamber (Conviron). Plants were then transferred to $4^{\circ} \mathrm{C}$ for a cold treatment, with no change in other growing parameters. Samples were taken from leaves and crowns before the beginning of the cold treatment (control), in the end of the 1st day (coldshock) and after 21 days of cold (acclimation). Samples collected from three biological replicates were used for phytohormone determination, protein analysis and quantitation of gene expression.

\section{Determination of Gene Expression by Quantitative RT-PCR}

Total RNA was isolated using the Direct-zol ${ }^{\mathrm{TM}}$ RNA Miniprep Kit (Zymo Research) according to the manufacturer's instructions. RNA was reverse transcribed by M-MLV reverse transcriptase and Oligo $(\mathrm{dT})_{18}$ primer (both from Thermo Scientific) according to the protocol of the manufacturer. Realtime quantitative PCR was performed using a CFX96 Touch ${ }^{\mathrm{TM}}$ Real-Time PCR Detection System (Bio-Rad) and gene-specific primers (Supplementary Table 1). Data analysis was performed as described by Kalapos et al. (2016). The relative quantities of the individual transcripts were calculated by the $\Delta \Delta C$ t method using a phosphogluconate-dehydrogenase gene (UniGene ID: Ta30797) for normalization (Paolacci et al., 2009). The relative expression values (fold change) were converted to $\log 2$ values, clustered and visualized with the Gitools software (Perez-Llamas and Lopez-Bigas, 2011). Ranking of genes was defined with the following formula: relative change $=$ ABS \{AVERAGE [Cheyenne + Chinese Spring(Cheyenne 5A)] - Chinese Spring\}.

\section{Hormone Analysis}

Leaf and crown samples (ca $50 \mathrm{mg} \mathrm{FW}$ ) were purified and analyzed according to Dobrev and Kamínek (2002) and Dobrev and Vankova (2012). Briefly, homogenized samples were extracted with cold methanol/water/formic acid (15/4/1 v/v/v). The following labeled internal standards ( $10 \mathrm{pmol} / \mathrm{sample})$ were added: ${ }^{2} \mathrm{H}_{3}$-PA (phaseic acid), ${ }^{2} \mathrm{H}_{3}$-DPA (dihydrophaseic acid), ${ }^{2} \mathrm{H}_{4}-7 \mathrm{OH}-\mathrm{ABA},{ }^{2} \mathrm{H}_{5}$-ABA-GE (ABA-glucosyl ester) (NRCPBI), ${ }^{2} \mathrm{H}_{6}$-ABA, ${ }^{2} \mathrm{H}_{5}$-transZ, ${ }^{2} \mathrm{H}_{5}$-transZR, ${ }^{2} \mathrm{H}_{5}$-transZ7G, ${ }^{2} \mathrm{H}_{5}$ transZ9G, ${ }^{2} \mathrm{H}_{5}$-transZOG, ${ }^{2} \mathrm{H}_{5}$-transZROG, ${ }^{2} \mathrm{H}_{5}$-transZRMP, ${ }^{2} \mathrm{H}_{3}$-DHZ, ${ }^{2} \mathrm{H}_{3}$-DHZR, ${ }^{2} \mathrm{H}_{3}$-DZRMP, ${ }^{2} \mathrm{H}_{7}$-DZOG, ${ }^{2} \mathrm{H}_{3}$-DHZ9G, ${ }^{2} \mathrm{H}_{7}$-DZOG, ${ }^{2} \mathrm{H}_{6}$-iP, ${ }^{2} \mathrm{H}_{6}$-iPR, ${ }^{2} \mathrm{H}_{6}$-iP7G, ${ }^{2} \mathrm{H}_{6}$ - iP9G, ${ }^{2} \mathrm{H}_{6}$-iPRMP (Olchemim). Extracts were purified using a mixed mode reverse phase-cation exchange SPE column (Oasis-MCX, Waters). ABA metabolites were eluted with methanol and CK metabolites with $0.35 \mathrm{M} \mathrm{NH}_{4} \mathrm{OH}$ in $60 \%$ methanol. Hormones were analyzed using HPLC (Ultimate 3000, Dionex) coupled to a hybrid triple quadrupole/linear ion trap mass spectrometer (3200 Q TRAP, Applied Biosystems). Three biological replicates were analyzed. The cold-treated/control ratios of measured phytohormones were converted to $\log 2$ values, clustered and visualized with the Gitools software (Perez-Llamas and Lopez-Bigas, 2011). Ranking of phytohormones was defined with the following formula: relative change $=\mathrm{ABS}$ \{AVERAGE $[$ Cheyenne + Chinese Spring(Cheyenne 5A)] - Chinese Spring\}.

\section{Determination of WCS120 Protein Content}

One gram (FW) of leaves or crowns were homogenized under liquid nitrogen with $4.5 \mathrm{~mL}$ extraction buffer $(100 \mathrm{mM}$ Tris$\mathrm{HCl}, \mathrm{pH} 8.8$, containing "Complete EDTA-free Protease Inhibitor Cocktail Tablets," Roche) using the mortar and pestle. The mixture was centrifuged twice at $20,000 \mathrm{~g}$ at $4^{\circ} \mathrm{C}$ for $20 \mathrm{~min}$, then kept in boiling water for $15 \mathrm{~min}$, cooled rapidly on ice and centrifuged at $20,000 \mathrm{~g}\left(4^{\circ} \mathrm{C}\right)$ for $20 \mathrm{~min}$. Concentration of heat-stable proteins was determined according to Bradford (1976). The supernatants were then precipitated by cold acetone with $1 \% 2$-mercaptoethanol $(\mathrm{v} / \mathrm{v})$ using 1:5 sample:acetone ratio $(\mathrm{v} / \mathrm{v})$. The pellet was then centrifuged at $20,000 \mathrm{~g}\left(4^{\circ} \mathrm{C}\right)$ for $20 \mathrm{~min}$ and dried. Dry samples were resolved in Laemmli Sample buffer prepared according to the Bio-Rad manual (Hercules). The sample equivalent to $0.4 \mathrm{mg}$ of tissue $\mathrm{FW}$ per genotype were loaded on $10 \%$ sodium dodecyl sulfate polyacrylamide gel (SDS-PAGE) (Laemmli, 1970). For the estimation of the protein molecular weight, "Precision Plus Protein ${ }^{\mathrm{TM}}$ Standards, All Blue" (Bio-Rad) were used.

Protein gel blots were carried out on a semi-dry blotter (GE Healthcare) for $55 \mathrm{~min}$ at $0.8 \mathrm{~mA} \mathrm{~cm}{ }^{-2}$ using a nitrocellulose membrane (pore diameter $0.45 \mu \mathrm{m}$; Bio-Rad). For hybridization with the antibody against dehydrin K-segment (Enzo Life Sciences, Inc.) to visualize dehydrins, an Immune-Blot Assay kit with Goat Anti-Rabbit IgG Alkaline Phosphatase (AP; Bio-Rad, Hercules) was used according to the manufacturer's instructions. Membranes with visualized dehydrins were scanned by a calibrated densitometer GS-800 (Bio-Rad) at $600 \mathrm{dpi}$. Determination of the relative DHN protein accumulation was carried out using the Quantity One 4.6.7 program (Bio-Rad). Gels stained by Bio-Safe Coomassie (Bio-Rad, Hercules) were used for normalization of densities of dehydrin bends to quantity the loaded proteins (Figure 1).

\section{RESULTS}

\section{Expression of Genes Related to Cold Acclimation}

In order to characterize the differences in cold stress responses among the studied genotypes and to prove the role of the chromosome 5A of Cheyenne in the improvement of freezing tolerance in the CS genetic background, expression profiles of four selected marker genes, VRN1 (Vernalization 1) (Figures 2A,B and Supplementary Table 2), CBF14 (C-repeat binding factor 14), Cor14b (Cold-responsive protein 14b) and 


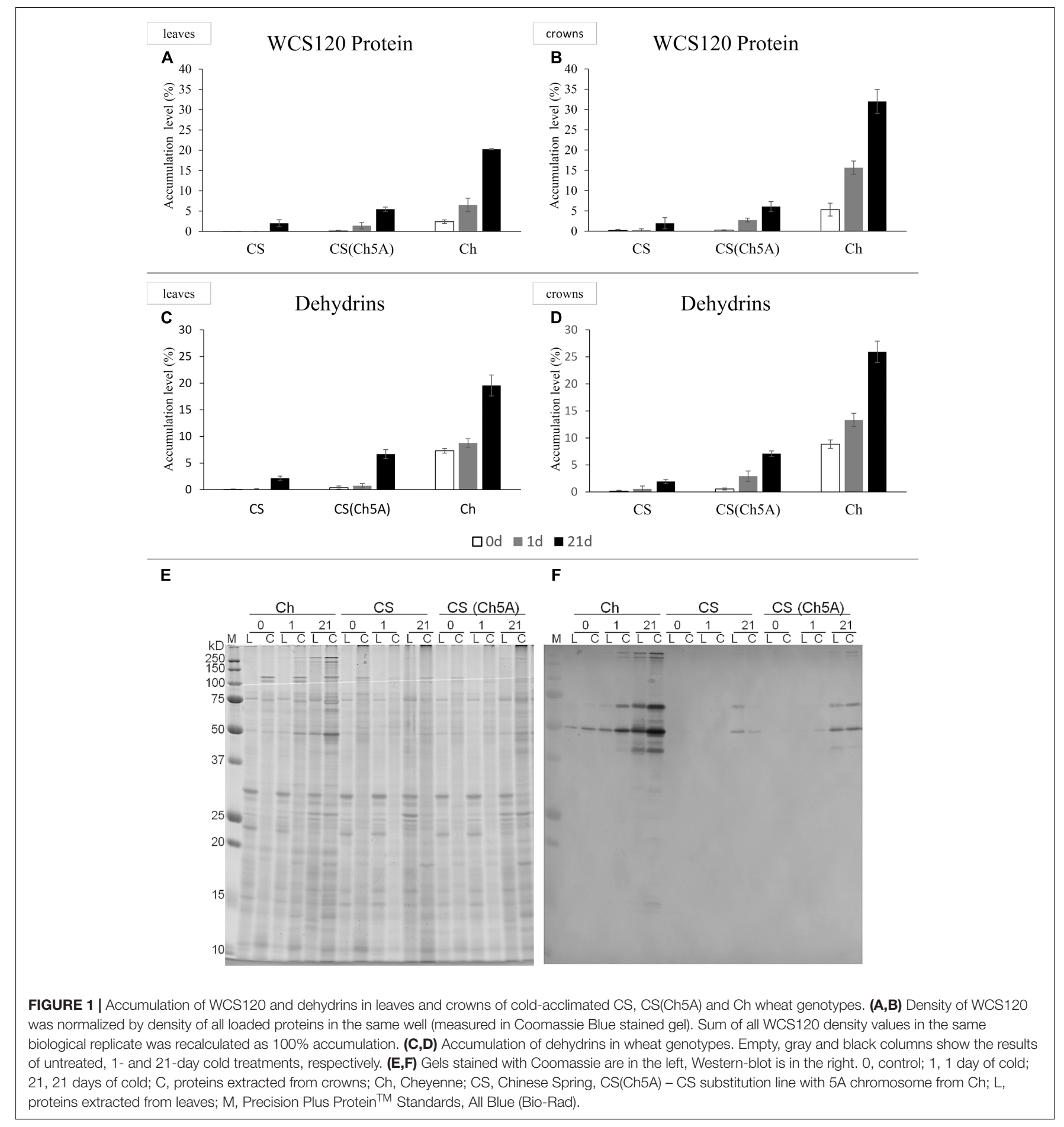

WCS120 (Wheat cold specific protein 120) were compared (Figures 2C,D and Supplementary Table 2).

After 1 day cold treatment, the expression of VRN1 did not change significantly in the leaves of either genotype. After 21 days, approximately 5- and 3-fold increase in VRN1 expression was detected in $\mathrm{CS}$ and $\mathrm{CS}(\mathrm{Ch} 5 \mathrm{~A})$ leaves, respectively, but only a low, non-significant elevation was detected in Cheyenne. Different expression patterns were observed in the crowns. In all examined genotypes, a slight increase of $V R N 1$ transcription was detected after 1 day, while about 2- to 4-fold VRN1 elevation was found after 21 days (Figures 2A,B and Supplementary Table 2). The VRN1 induction was stronger in CS than in the freezingtolerant genotypes.

After 1 day cold treatment (Kalapos et al., 2016), expression of CBF14 exhibited much higher increase in leaves of $\mathrm{Ch}$ and CS(Ch5A) genotypes than in CS (about 1000- and 500-fold, 
respectively, vs. about 70 -fold). Although expression of CBF14 decreased after 3 weeks of cold treatment in all genotypes, it remained still much higher in the freezing-tolerant genotypes than in CS (Figures 2C,D and Supplementary Table 2). In crowns, the expression of CBF14 increased in all genotypes after 1 day cold-shock. The increase in the crowns was, however, considerably lower than in the leaves (Kalapos et al., 2016), with the lowest increase in CS. During acclimation, further increase of CBF14 was observed, in CS comparable to that in leaves. The elevation in $\mathrm{Ch}$ and $\mathrm{CS}(\mathrm{Ch} 5 \mathrm{~A})$ was at least 4 times more profound than in CS.

After 1 day cold treatment, expression of the Cor $14 b$ highly increased in the leaves of all genotypes, especially in Ch. The transcript levels remained high only in the freezing-tolerant genotypes. The increase of transcript abundance was even higher in crowns, at least 600 times, 170 times and 100 times higher in $\mathrm{Ch}, \mathrm{CS}(\mathrm{Ch} 5 \mathrm{~A})$ and $\mathrm{CS}$, respectively, compared to the control. In the freezing-tolerant genotypes, the Cor14b transcript levels remained considerably higher than in the sensitive CS cultivar after 21 days (Figures 2C,D and Supplementary Table 2).

The amounts of WCS120 transcripts were close to the detection limit in the control samples. After 1 day cold treatment, expression of WCS120 increased almost 100-fold in the leaves of $\mathrm{CS}$, while it was, in comparison with CS, at least 10 times higher in both $\mathrm{Ch}$ and CS(Ch5A) (Figures 2C,D and Supplementary Table 2). Although WCS120 expression decreased after the 21-day treatment, the freezing-tolerant genotypes still contained 3- to 5 -fold more transcripts than the sensitive CS. The elevation of transcript levels was also high in the crowns, but the pattern was different. The high values reached after 1 day cold-shock in the freezing-tolerant genotypes were further elevated after the longterm cold treatment. Crowns of $\mathrm{Ch}$ and $\mathrm{CS}(\mathrm{Ch} 5 \mathrm{~A})$ genotypes exhibited about 10- to 20-fold more WCS120 transcripts than CS.

The level of WCS120 protein was generally in accordance with the gene expression pattern (Figures 1A,B). The freezingtolerant genotypes accumulated higher levels of WCS120 than $\mathrm{CS}$ in all treatments. Under the control conditions, the level of WCS120 was clearly detectable in Ch, in contrast to the negligible WCS120 accumulation in the other two genotypes. After 1 day of cold treatment, significant increase (about 4-fold) of WCS120 was found only in Ch crowns. In crowns of all genotypes, the amount of WCS120 was higher than in their leaves at both time points, except in CS after 21-day treatment. After 3 weeks of cold treatment, the level of WCS120 in all genotypes correlated with their freezing tolerance, i.e., the highest and lowest WCS120 accumulation was found in freezing-tolerant $\mathrm{Ch}$ and freezing-sensitive CS, respectively, while in the moderately freezing-tolerant CS(Ch5A) the level of WCS120 was in-between.

\section{Expression of Genes Related to Abscisic Acid}

In leaves, expression of ZEP (zeaxanthin epoxidase), which catalyzes an early step in ABA biosynthesis, increased after 1 day cold-shock (Kalapos et al., 2016), but decreased after 21 days of cold treatment in all genotypes (Figure 2E and Supplementary Table 2). The expression pattern correlated with the changes

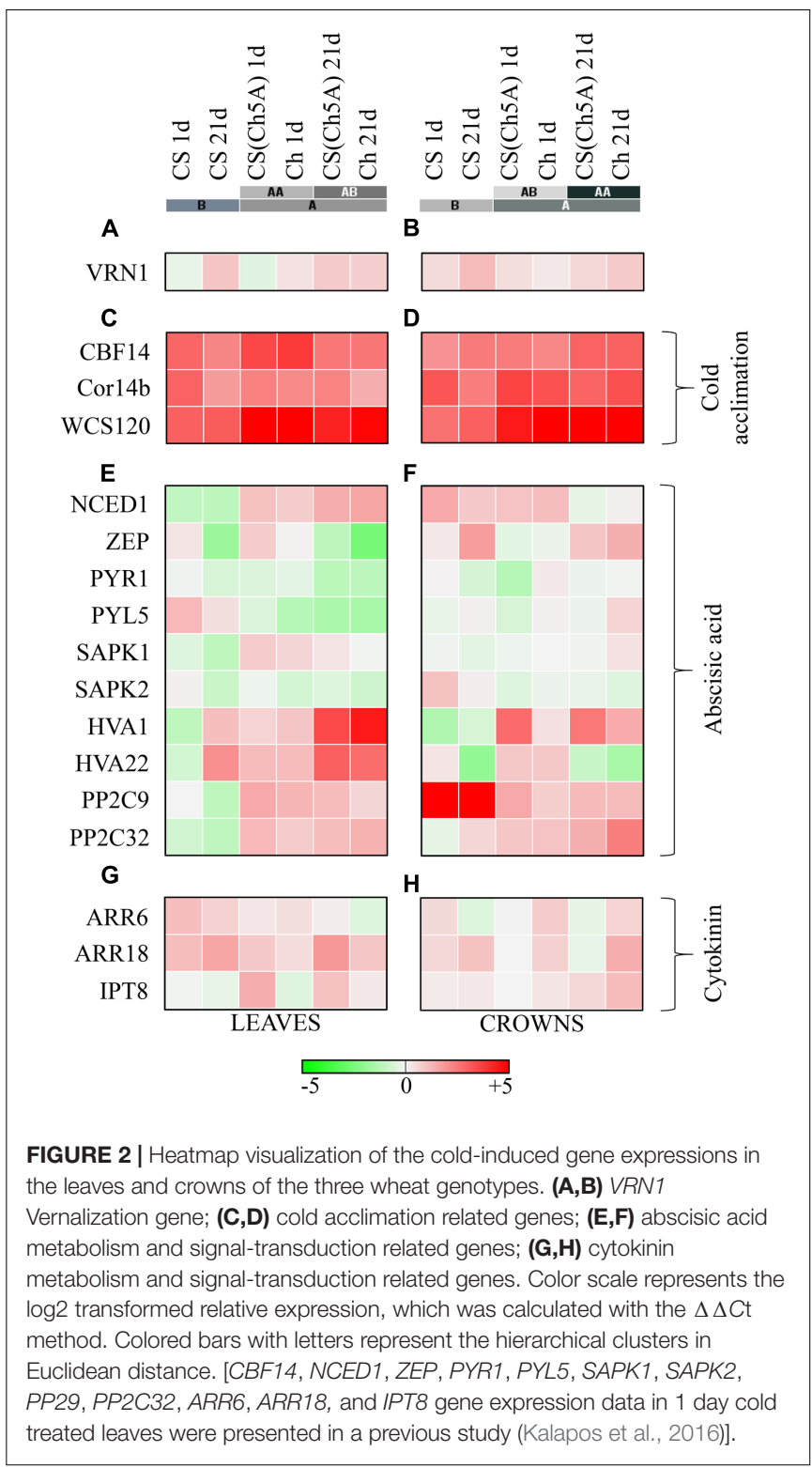

in ABA levels (see below). In contrast, the transcript level was changed in the opposite fashion in crowns (Figure 2F and Supplementary Table 2).

Expression of the NCED1 (9-cis-epoxycarotenoid dioxygenase), coding the rate limiting ABA biosynthetic enzyme, highly correlated with determined ABA levels, both in leaves and crowns. NCED1 expression was down-regulated in leaves of the freezing-sensitive CS variety during the whole cold treatment, while it was highly elevated in the freezing-tolerant genotypes (Figure 2E and Supplementary Table 2). In crowns, the transcript level was also up-regulated in all examined genotypes, but a slight reduction was detected in the freezing-tolerant genotypes after 21-day cold (Figure 2F and Supplementary Table 2).

Cold-shock down-regulated the expression of the ABA receptor gene PYR1 (Pyrabactin resistance 1) in leaves (Kalapos 
et al., 2016). Further suppression of PYR1 expression was observed after prolonged stress, especially in freezing-tolerant genotypes (Figure 2E and Supplementary Table 2). In crowns, such strong down-regulation was observed only in Ch (Figure $2 \mathrm{~F}$ and Supplementary Table 2).

The expression of PYL5 (PYR1-like protein 5) highly increased in the leaves of the freezing-sensitive variety CS (Kalapos et al., 2016), but decreased in the freezing-tolerant genotypes CS(Ch5A) and Ch (Figure 2E and Supplementary Table 2). In crowns, PYL5 transcription was slightly down-regulated after 1 day cold-shock, increasing by the 21 st day of the cold treatment in the leaves of all genotypes with the highest level measured in Ch variety (Figure 2F and Supplementary Table 2).

The SnRK2 kinase SAPK1 (Stress-activated protein kinase 1), a component of the ABA signal transduction pathway was strongly down-regulated by short- and long-term cold treatment in leaves of the freezing-sensitive genotype CS (Kalapos et al., 2016), and was up-regulated in the freezing-tolerant genotypes (Figure $2 \mathrm{E}$ and Supplementary Table 2). In crowns, changes in SAPK1 expression were not significant (Figure 2F and Supplementary Table 2).

Another serine/threonine-protein kinase gene, SAPK2 (Stressactivated protein kinase 2) was down-regulated in leaves of all genotypes after 1 day cold treatment (Kalapos et al., 2016), declining further after 21 days (Figure 2E and Supplementary Table 2). Crowns exhibited a similar tendency, however, the transcription of SAPK2 was stronger in CS compared to $\mathrm{CS}(\mathrm{Ch} 5 \mathrm{~A})$ and $\mathrm{Ch}$ (Figure 2F and Supplementary Table 2).

Expression of two ABA-inducible genes, HVA1 (ABAinducible protein HVA1) and HVA22 (ABA-inducible protein HVA22), was down-regulated in CS leaves, while up-regulated in both tolerant genotypes after 1 day cold treatment. Both genes were up-regulated in all genotypes after 21 days. In crowns, the HVA1 expression had the same pattern as in leaves. HVA22 expression was up-regulated in Ch and CS, but not CS(Ch5A) line during the whole cold period. This suggests that the two genes might have diverse spatial and temporal regulation associated with their different roles in each genotype (Figures 2E,F and Supplementary Table 2).

The genes for two 2C-type protein phosphatases involved in ABA signaling, PP2C9 (2C-type protein phosphatase protein C9) and PP2C32 (2C-type protein phosphatase protein C32), were similarly down- and up-regulated in leaves of freezing-sensitive and tolerant genotypes, respectively, both after 1 (Kalapos et al., 2016) and 21 days of cold (Figure 2F and Supplementary Table 1). In crowns, however, PP2C9 was strongly up-regulated in CS variety (at both time points). Expression of PP2C32 was only slightly enhanced in CS. Moderate up-regulation of the expression of both phosphatase genes was observed in freezingtolerant genotypes (Figure 2E and Supplementary Table 2).

\section{Expression of Genes Related to Cytokinins}

The expression of a CK biosynthetic gene, IPT8 (isopentenyl transferase 8 ), was up-regulated during the cold-shock in leaves of the substitution line (Kalapos et al., 2016) (Figure 2G and
Supplementary Table 2). In crowns, moderate up-regulation of IPT8 was observed in freezing-tolerant genotypes after the longterm cold (Figure $\mathbf{2} \mathbf{H}$ and Supplementary Table 2 ).

Expression of the negative, type-A response regulator ARR6 was elevated by 1 day cold-shock in leaves (Kalapos et al., 2016), most strongly in CS. During acclimation, ARR6 expression decreased, especially in Ch. Similar profile was observed in crowns, with exception of $\mathrm{Ch}$, which maintained elevated ARR6 expression even after 21 days.

Expression of the positive signaling component, type-B response regulator $A R R 18$ (transcription factor) was mildly increased in the leaves of all genotypes after 1 day cold (Kalapos et al., 2016) and strongly increased after 21-day stress (in Ch decreased). This tendency was detected also in crowns, with exception of CS(Ch5A), where no significant up-regulation was found.

\section{Dynamics of Abscisic Acid and Cytokinin Levels}

In order to evaluate the impact of the substitution of the $5 \mathrm{~A}$ chromosome of the spring wheat Chinese Spring by the $5 \mathrm{~A}$ chromosome from the winter wheat Cheyenne, the hormone levels were measured after 1 day cold-shock (Kalapos et al., 2016) and 21-day acclimation period. The analyzed hormones included $\mathrm{ABA}$ and $\mathrm{CK}$ metabolites.

The ABA increase was most profound in $\mathrm{Ch}$ leaves and crowns after 1 day cold (Figures 3A,B and Supplementary Table 3). The ABA elevation in CS(Ch5A) line was lower, while only moderate increase was found in CS leaves. Cold stress was generally associated with the decrease of ABA inactive metabolites phaseic acid (PA) and neophaseic acid (NeoPA) in leaves of all genotypes. PA increased only in CS and CS(Ch5A) crowns during prolonged stress. Dihydrophaseic acid (DPA) and ABA-glucosyl ester (ABA-GE) generally decreased in cold in all genotypes and tissues (Figures 3A,B and Supplementary Table 3).

The active $\mathrm{CKs}$ include trans-zeatin ( $\mathrm{tZ}$ ), isopentenyladenine (iP), dihydrozeatin (DHZ) and cis-zeatin (cZ). Cold-shock was associated with down-regulation of active CKs in leaves of the winter variety $\mathrm{Ch}$ (Kalapos et al., 2016), especially of $\mathrm{tZ}$ and DHZ. The level of $\mathrm{CZ}$ (CK with relatively low physiological activity, usually associated with growth-limiting conditions), however, was transiently increased. In contrast, the spring variety CS exhibited during cold-shock up-regulation of active CKs, including strong increase of $\mathrm{cZ}$ and DHZ. The CS(Ch5A) line exhibited intermediate response in leaves, cold-shock did not impose significant change in iP and only mild increase in $\mathrm{tZ}$. As the level of $c Z$ was down-regulated, the total amount of active CKs decreased. During acclimation, $\mathrm{tZ}$ and iP increased in leaves of all genotypes, which may indicate re-establishment of growth, however, cZ strongly decreased in comparison with control conditions (Figure 3C and Supplementary Table 3).

In crowns, mild decrease and no significant change of iP and $\mathrm{tZ}$ levels, respectively, were observed in $\mathrm{Ch}$ and $\mathrm{CS}(\mathrm{Ch} 5 \mathrm{~A})$ genotypes. The levels of $c Z$ were strongly down-regulated. Only spring variety CS exhibited substantial elevation of total 


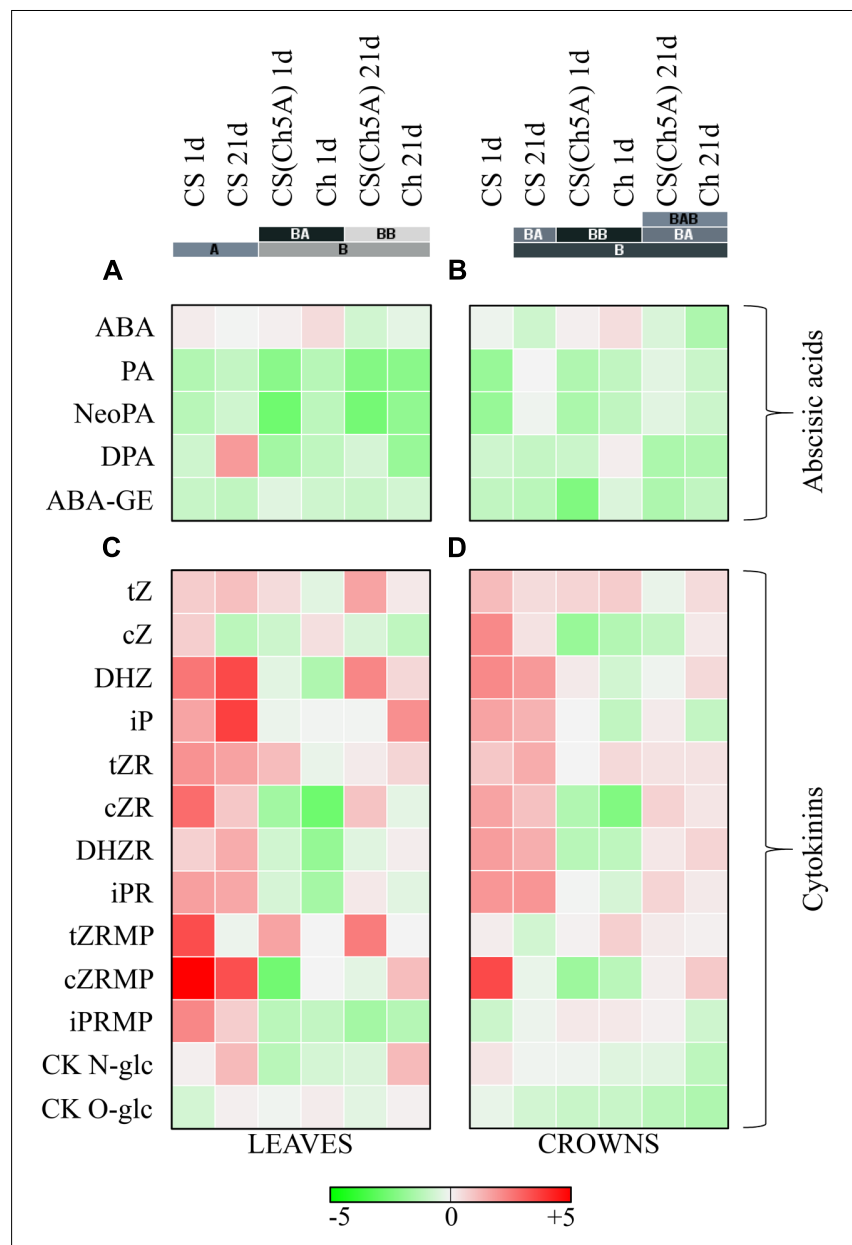

FIGURE 3 | Heatmap visualization of the cold induced phytohormone levels in leaves and crowns of the three wheat genotypes. (A,B) Abscisic acid metabolites: ABA, abscisic acid; PA, phaseic acid; NeoPA, neophaseic acid; DPA, dihydrophaseic acid; ABA-GE, abscisic acid-glucosyl ester. (C,D) Cytokinin metabolites: $\mathrm{ZZ}$, Trans-zeatin; $\mathrm{CZ}$, cis-zeatin; DHZ, Dihydrozeatin; iP, Isopentenyladenine; tZR; Trans-zeatin riboside; cZR, cis-zeatin riboside; $\mathrm{DHZR}$, dihydrozeatin riboside; iPR, isopentenyladenosine; tZRMP, Trans-zeatin riboside monophosphate; cZRMP, cis-zeatin riboside monophosphate; iPRMP, isopentenyladenosine monophosphate; $\mathrm{CK} \mathrm{N}$-glc, cytokinin $\mathrm{N}$-glucosides; CK O-glc, cytokinin O-glucosides. Color scale represents the log2 transformed cold treatment/control ratio of hormone levels. Colored bars with letters represent the hierarchical clusters in Euclidean distance. [Phytohormone accumulation data in 1 day cold treated leaves were presented in a previous study (Kalapos et al., 2016)].

active CKs (especially during cold-shock) (Figure 3D and Supplementary Table 3).

Temperature decrease seems to be associated with the suppression of $\mathrm{CK}$ biosynthesis in $\mathrm{Ch}$ and $\mathrm{CS}(\mathrm{Ch} 5 \mathrm{~A})$ leaves, as indicated by decrease of precursors of active CKs CK phosphates. In contrast, in CS leaves, highly significant up-regulation of $\mathrm{CK}$ phosphates was found, given mainly by elevation of cZRMP, which coincided with elevation of active CKs. After 21 days of cold, CK phosphates in the frost-tolerant genotypes [Ch and $\mathrm{CS}(\mathrm{Ch} 5 \mathrm{~A})]$ were still very low, while CS variety exhibited slightly higher level than the corresponding control plants (Figure 3C and Supplementary Table 3). In crowns, as meristematic tissues, CK phosphates were much more abundant than in leaves. No significant change in total CK phosphates was detected upon cold-shock in frost-tolerant genotypes. In case of CS, high increase of cZRMP was detected after 1 day cold (Figure 3D and Supplementary Table 3).

The CK ribosides represent transport forms, which are easily convertible into the active forms (bases). Frost-tolerant genotypes exhibited upon cold-shock decrease of CK ribosides in leaves, in contrast to CS, which substantially increased CK riboside levels, especially of cZR (Kalapos et al., 2016). After prolonged cold stress, $\mathrm{Ch}$ reached the control levels, the substitution line showed moderate elevation above the original value, while CS showed the highest increase. In crowns, coldshock was associated with decrease of cZR only in frost-tolerant genotypes $\mathrm{Ch}$ and $\mathrm{CS}(\mathrm{Ch} 5 \mathrm{~A})$ (Figures 3C,D and Supplementary Table 3).

Down-regulation of $\mathrm{CK}$ biosynthesis in leaves of $\mathrm{Ch}$ and $\mathrm{CS}(\mathrm{Ch} 5 \mathrm{~A})$ genotypes was during cold-shock accompanied by decrease of CK deactivation by CK $\mathrm{N}$-glucosylation (Kalapos et al., 2016). During acclimation, when active CKs were up-regulated, the levels of $\mathrm{CK} N$-glucosides in leaves increased, probably in order to fine-regulate the $\mathrm{CK}$ pool. In crowns, gradual decrease of $\mathrm{CK} N$-glucosides was found in $\mathrm{Ch}$, mild up-regulation being found in CS. In contrast, CK O-glucosylation was upon cold-shock promoted predominantly in crowns of the spring variety CS, in leaves only after prolonged cold stress (Figures 3C,D and Supplementary Table 3).

\section{DISCUSSION}

The CBF regulon situated on the 5th chromosome of cereals has been known to play a pivotal role in the cold stress response in plants. We tested the impact of $5 \mathrm{~A}$ chromosome on the cold reactions and freezing tolerance in wheat using the single chromosome substitution system. Since CBF genes, apart from the regulation of the Cor effector genes, also affect the hormonal homeostasis (Achard et al., 2008; Soltész et al., 2013), this substitution line provides a unique tool to elucidate the hormonal basis of effective cold acclimation. The effect of the $5 \mathrm{~A}$ chromosome substitution on frost tolerance was reported to be associated with the function of the vernalization gene VRN1, which was mapped $\sim 30 \mathrm{cM}$ apart from the $F r-2$ on the long arms of homologous group 5 chromosomes (Vágújfalvi et al., $2000,2003,2005)$. However, in our genetic system the expression profile of VRN1 in CS and CS(Ch5A) was quite similar, both in leaves and crowns. So, the observed higher expression values of three freezing tolerance marker genes (CBF14, Cor14b, and WCS120) found in CS(Ch5A) lines were most likely due to the presence of Ch5A Fr-2 locus in the CS genetic background and the potential modifying effect of VRN1 was negligible. The expression values of the stress marker genes measured in $\mathrm{CS}(\mathrm{Ch} 5 \mathrm{~A})$ line were in between $\mathrm{CS}$ and $\mathrm{Ch}$ ones. The reason might be that apart from the $5 \mathrm{~A}$, also the $5 \mathrm{D}$ and to lesser extent $5 \mathrm{~B}$ chromosomes are also effective in the performance of freezing tolerance, when originated from winter habit wheats 
(Sutka, 1981; Veisz and Sutka, 1989; Tóth et al., 2003; Pearce et al., 2013).

Comparing the tissue specific expression pattern of CBF14 transcription factor, it is interesting to mention that although the expression level was considerably higher in leaves than in crowns after 1 day cold treatment, in the acclimation phase the transcript levels decreased in leaves, while further significantly increased in crowns. The expression level both in $\mathrm{Ch}$ and $\mathrm{CS}(\mathrm{Ch} 5 \mathrm{~A})$ was at every tested time point significantly higher than in CS. Thus, CBF14 expression can be a good marker for freezing tolerance both in leaves and crowns during the cold stress response. In the case of the effector genes, the transcript level of WCS120 seems to be much more useful positive marker of freezing tolerance than that of Cor14b. Independently from the treatment period, the WCS120 transcript levels were significantly higher both in $\mathrm{Ch}$ and $\mathrm{CS}(\mathrm{Ch} 5 \mathrm{~A})$ genotypes than in CS. Although the expression level of Cor14b was significantly higher in Ch leaves and crowns in comparison with CS during the whole tested period, in case of CS(Ch5A) the Cor14b pattern was different. The Cor14b transcript level in the CS(Ch5A) crowns was higher after 1 day cold treatment, while in leaves it was higher after 21 days. In contrast to the transcript pattern of WCS120, which was very similar to that of CBF14, the Cor $14 b$ dynamics differed significantly. The reason might be that the Cor $14 b$ is regulated not only by CBF genes, but also by light (Crosatti et al., 1999).

\section{ABA Content and Expression of ABA Biosynthesis Genes}

Temperature decrease is associated with the drop of the root hydraulic conductivity. The response to the resulting water deficit includes transient elevation of ABA. Short-term increase of ABA content was observed in leaves and crowns of all genotypes, most strongly in Ch. This transient ABA elevation is in accordance with previous studies on the hormonal responses to cold stress (Kosová et al., 2012; Vanková et al., 2014). Transient ABA up-regulation coincided with the elevation of the expression of gene coding for the rate limiting enzyme of ABA biosynthesis, NCED1. After cold-shock, NCED1 expression was stimulated in crowns of all genotypes and in leaves of freezing-tolerant ones (Kalapos et al., 2016), where it was preserved during the whole stress treatment. In these genotypes, down-regulation of ABA levels after prolonged cold coincided in crowns with suppression of NCED1 expression. The leaf samples of CS variety did not show difference between the short (Kalapos et al., 2016) and long-term cold treatments. Our data are in accordance with Wu et al. (2014), who found high increase of NCED1 during cold acclimation (at $4^{\circ} \mathrm{C}$ ) in grapevine. Stimulation of NCED expression was reported in case of several abiotic stresses, not only by cold. The up-regulation of AtNCED was found in drought-stressed Arabidopsis plants, where it was associated with the induced ABA levels (Xiong et al., 2002). PaNCED1 from avocado was highly expressed in leaves after dehydration (Chernys and Zeevaart, 2000). Transient expression of CrNCED1 in tobacco led to an increase in the ABA level as well as to enhanced tolerance to multiple abiotic stresses (Xian et al., 2014).

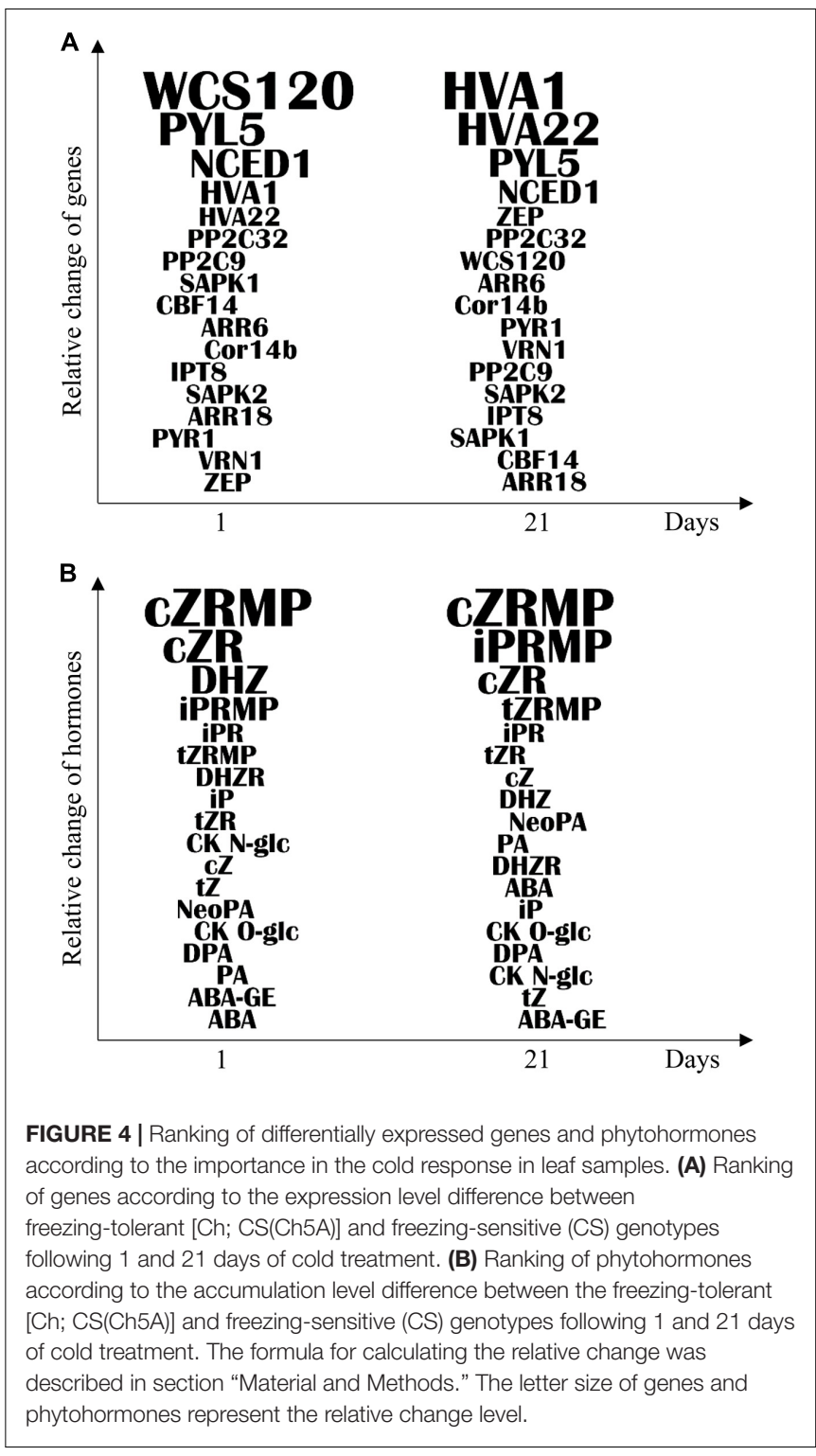

Expression of another ABA biosynthesis related gene, ZEP, displayed a different pattern in leaves and crowns. Transcription of $Z E P$ was up-regulated in leaves upon cold-shock (Kalapos et al., 2016), while it was strongly enhanced in crowns after prolonged cold treatment (in all genotypes). This agrees with the report of Xiong et al. (2002), who found no up-regulation of ZEP expression in leaves of Arabidopsis plants after longer cold treatment. In Medicago sativa leaves too the expression of MsZEP considerably fell during the long-term cold treatment (Zhang H. et al., 2016). Our results revealed that the cold treatment-dependent down-regulation of ZEP in leaves and its up-regulation in crowns are associated with the organ-specific role in frost tolerance.

\section{ABA Signaling and Inducible Genes}

The ABA signal transduction starts with the formation of a complex between ABA, PYR/PYL receptor protein and PP2C 
(repressor), which leads to the inhibition of PP2C activity and its degradation, resulting in the activation of SnRK2s. The activated SnRK2s then activate different downstream proteins, such as transcription factors and ion channels, which subsequently trigger ABA responses (Park et al., 2009; Gonzalez-Guzman et al., 2012). In our study, we followed expression of three core components of ABA signaling system - receptor genes (PYR1, PYL5), protein phosphatases 2C (PP2C9, PP2C32), the SnRK2 kinases (SAPK1 and SAPK2) and two ABA-inducible genes coding LEA proteins (HVA1, HVA22). The expression of two ABA receptors, PYR1 and PYL5, was strongly downregulated in the leaves in cold and only slight enhancement was observed in the crowns of the freezing-tolerant genotypes. The expression of SAPK2 was down-regulated in the freezingsensitive leaves, and was up-regulated in the cold-shocked CS crowns. In parallel to this, the $2 \mathrm{C}$-type protein phosphatases, PP2C9 and PP2C32, were markedly up-regulated. These findings suggest that the suppression of ABA signaling, including the effect on ion channels and subsequently on stomata aperture, occurs in freezing-tolerant genotypes, especially after prolonged cold. However, it should be taken into consideration that the regulation of phosphatase and kinase activities is given by their phosphorylation/dephosphorylation, thus the expression pattern may not correlate precisely with their activities. From this point of view, relevant information may be obtained from the expression pattern of ABA-inducible genes. The up-regulation of genes coding LEA proteins, HVA1 and HVA22, was delayed in CS leaves, being rapidly stimulated by cold-shock and further enhanced after prolonged stress in freezing-tolerant genotypes. In crowns, the expression was also higher in freezing-tolerant genotypes. These data suggest that ABA function in regulation of stomata aperture is important especially after the cold-shock. Later on, the effect of ABA on stimulation of the expression of protective proteins persists. This assumption is in accordance with the reports on positive effects of strengthened ABA signal transduction on the abiotic stress tolerance. Overexpression of PYR/PYL genes, such as PYR1 and PYL5, enhanced the response to $\mathrm{ABA}$ in Arabidopsis plants and enhanced drought tolerance in rice (Santiago et al., 2009; Kim et al., 2014). TaSnRK2s were generally up-regulated by $\mathrm{NaCl}$ and cold treatment, with a maximal expression level at $6 \mathrm{~h}$ of the stress or later (Zhang Z. et al., 2016). TaSnRK2C1 overexpression in transgenic tobacco increased the tolerance against dehydration, salt and low temperature (Du et al., 2013). Overexpression of other SnRK2s, such as TaSNRK2.3, TaSnRK2.4, TaSnRK2.7, and TaSnRK2.8 resulted in enhanced tolerance against drought, salt, and low temperature (Mao et al., 2010; Zhang et al., 2010, 2011; Tian et al., 2013).

Special attention has been paid to ABA-inducible LEA (Late Embryogenesis Abundant) genes. These genes were detected in high abundance in the wheat dormant seeds (Sutton et al., 1992). HVA1 mRNA was also highly accumulated after 6 h cold treatment in two freezing-resistant barley cultivars (Ried and Walker-Simmons, 1993). Overexpression of HVA1 in transgenic rice significantly elevated the tolerance against water deficit and salt stress (Xu et al., 1996). In transgenic rice, HVA1 was highly accumulated in root apical meristem and lateral root primordia, enhancing the tolerance to drought, salt and cold stresses (Chen et al., 2015). The barley HVA1 overexpression in transgenic mulberry increased the drought, salt, and cold tolerance (Checker et al., 2012). Another LEA gene, HVA22, was directly induced by dehydration and cold stress both in shoots and roots of 3-day barley seedlings (Shen et al., 2001). HVA22 was also rapidly induced by ABA and cycloheximide (Shen et al., 1993). Our results accord with the above mentioned studies and suggest that LEA genes, including HVA1 and HVA22, play an important role during cold acclimation of wheat.

\section{Cytokinin Levels and Cytokinin- Related Genes}

Cytokinins, phytohormones associated predominantly with stimulation of cell division and growth, have been recognized to play important roles in the stress responses, too (Ha et al., 2012).

Cytokinin's growth promoting functions require dynamic and precise regulation of their levels and signal transduction during the cold stress progression. Application of cold-shock is accompanied with fast down-regulation of $\mathrm{CK}$ levels to allow growth suppression and re-allocation of energy sources from growth to the defense. Later on, during acclimation, CKs participate in growth re-initiation, exhibiting also positive effect on photosynthesis. In accordance with these hypotheses, active CKs (tZ, cZ, DHZ, and iP) in leaves and crowns of freezing-tolerant genotypes were decreased after cold-shock. This suppression was not observed in sensitive CS genotype (Kalapos et al., 2016). The expression of the negative, type-A response regulator, $A R R 6$, was enhanced upon cold-shock in leaves (Kalapos et al., 2016) and crowns of all genotypes. This is in accordance with the reports of Vogel et al. (2005) and Jeon et al. (2010), who found a fast transient up-regulation of type-A response regulators, including $A R R 6$, upon cold stress. Higher transcript level of ARR6 in the leaves of cold-shocked freezingsensitive CS genotype suggests that the function of ARR6 is predominantly in regulation of CK signaling and rather indirectly in the cold stress response.

During acclimation, the levels of active CKs moderately increased. The expression of ARR6 was down-regulated, in leaves predominantly in $\mathrm{Ch}$, in crowns in CS and substitution line. Simultaneously, the expression of the positive, type-B response regulator, $A R R 18$, was enhanced, to higher extent in leaves of CS and substitution line and in crowns in all genotypes (mostly in Ch). Elevation of active CK levels as well as their signal transduction indicates at least partial acclimation to the new environmental conditions, especially in freezing-tolerant genotypes. The importance of up-regulation of CK signaling pathway is indicated by the fact that loss-of-function ARR6 mutants (i.e., mutants with promoted CK signal transduction) showed enhanced freezing tolerance in Arabidopsis (Jeon et al., 2010). CK profile during the cold stress progression is in accordance with reports of Kosová et al. (2012) and Vanková et al. (2014).

The key CK biosynthetic enzyme is isopentenyl transferase. In our experiments, we determined expression of IPT8. Surprisingly, it was enhanced in leaves of the substitution line and to a mild 
extent in crowns of all genotypes. The reason for the discrepancy between the profiles of active CKs and IPT8 expression might be the presence of multiple genes for CK biosynthesis, each regulated in a specific way (there are 9 isogenes in Arabidopsis).

Our findings suggest that the expression of type-A ARR6 and type-B ARR18 response regulators seems not to be under the control of chromosome 5A. These two genes are similarly regulated in the $\mathrm{CS}(\mathrm{Ch} 5 \mathrm{~A})$ substitution line and in the genetic background genotype CS.

\section{CONCLUSION}

Evaluation of the role of chromosome 5 in freezing tolerance and regulation of hormone stress responses was performed comparing freezing-sensitive and freezing-tolerant genotypes with $\mathrm{CS}(\mathrm{Ch} 5 \mathrm{~A})$ substitution line. Based on our results we conclude that wheat chromosome $5 \mathrm{~A}$ regulates, both directly and indirectly, the establishment of freezing tolerance. Apart of the already known effect on major cold inducible genes, including CBF14, Cor14b, and WCS120, we found that chromosome $5 \mathrm{~A}$ is associated also with regulation of hormone-related genes and metabolites underlying the freezing tolerance. Using a systems biological approach, dynamics of the expression of freezing tolerance marker genes was correlated with the changes in phytohormone contents, as well as with transcription pattern of the hormone related genes. The most relevant differences between the two tolerant genotypes and CS may become useful as molecular markers of freezing tolerance (Figures 4A,B).

\section{REFERENCES}

Achard, P., Gong, F., Cheminant, S., Alioua, M., Hedden, P., and Genschik, P. (2008). The cold-inducible CBF1 factor-dependent signaling pathway modulates the accumulation of the growth-repressing DELLA proteins via its effect on gibberellin metabolism. Plant Cell 20, 2117-2129. doi: 10.1105/tpc.108.058941

Boldizsár, Á., Carrera, D. Á., Gulyás, Z., Vashegyi, I., Novák, A., Kalapos, B., et al. (2016). Comparison of redox and gene expression changes during vegetative/generative transition in the crowns and leaves of chromosome $5 \mathrm{~A}$ substitution lines of wheat under low-temperature condition. J. Appl. Genet. 57, 1-13. doi: 10.1007/s13353-015-0297-2

Bradford, M. M. (1976). A rapid and sensitive method for the quantitation of microgram quantities of protein utilizing the principle of protein-dye binding. Anal. Biochem. 72, 248-254. doi: 10.1016/0003-2697(76)90527-3

Cahalan, C., and Law, C. N. (1979). The genetical control of cold resistance and vernalisation requirement in wheat. Heredity (Edinb). 42, 125-132. doi: 10.1038/hdy.1979.16

Campoli, C., Matus-Cádiz, M. A., Pozniak, C. J., Cattivelli, L., and Fowler, D. B. (2009). Comparative expression of Cbf genes in the Triticeae under different acclimation induction temperatures. Mol. Genet. Genomics 282, 141-152. doi: 10.1007/s00438-009-0451-9

Cattivelli, L., Baldi, P., Crosatti, C., Di Fonzo, N., Faccioli, P., Grossi, M., et al. (2002). Chromosome regions and stressed-realted sequences involved in resitance to abiotic stress in Triticeae. Plant Mol. Biol. 48, 649-665. doi: 10.1023/A:101482440

Checker, V. G., Chhibbar, A. K., and Khurana, P. (2012). Stress-inducible expression of barley Hval gene in transgenic mulberry displays enhanced tolerance against drought, salinity and cold stress. Transgenic Res. 21, 939-957. doi: 10.1007/s11248-011-9577-8

\section{AUTHOR CONTRIBUTIONS}

BK performed the gene expression experiments, analyzed the data, and wrote the manuscript. AN participated in data analysis. PD and RV performed hormone measurements. PV performed protein analysis. FM participated in data analysis. GG and RV conceived the study and participated in writing of the manuscript.

\section{FUNDING}

This work was supported by the Hungarian Research Fund (OTKA K111879), by Czech Science Foundation (17-06613S), by Czech Ministry of Agriculture (RO0416 and QJ1530373) and by Czech Ministry of Education, Youth and Sports (LD15167 as a part of COST action FA1306).

\section{ACKNOWLEDGMENT}

The authors wish to thank A. Horváth and M. Fehér for their help in plant cultivation and treatment.

\section{SUPPLEMENTARY MATERIAL}

The Supplementary Material for this article can be found online at: https://www.frontiersin.org/articles/10.3389/fpls.2017.02033/ full\#supplementary-material

Chen, Y., Carver, B. F., Wang, S., Zhang, F., and Yan, L. (2009). Genetic loci associated with stem elongation and winter dormancy release in wheat. Theor. Appl. Genet. 118, 881-889. doi: 10.1007/s00122-008-0946-5

Chen, Y. S., Lo, S. F., Sun, P. K., Lu, C. A., Ho, T. H. D., and Yu, S. M. (2015). A late embryogenesis abundant protein HVA1 regulated by an inducible promoter enhances root growth and abiotic stress tolerance in rice without yield penalty. Plant Biotechnol. J. 13, 105-116. doi: 10.1111/pbi.12241

Chernys, J. T., and Zeevaart, J. A. (2000). Characterization of the 9-cisepoxycarotenoid dioxygenase gene family and the regulation of abscisic acid biosynthesis in avocado. Plant Physiol. 124, 343-353. doi: 10.1104/pp.124. 1.343

Crosatti, C., Marè, C., Mazzucotelli, E., Belloni, S., Barilli, S., Bassi, R., et al. (2003). Genetic analysis of the expression of the cold-regulated gene cor14b: a way toward the identification of components of the cold response signal transduction in Triticeae. Can. J. Bot. 81, 1162-1167. doi: 10.1139/b03-114

Crosatti, C., Nevo, E., Stanca, A. M., and Cattivelli, L. (1996). Genetic analysis of the accumulation of COR 14 proteins in wild (Hordeum spontaneum) and cultivated (Hordeum vulgare) barley. Theor. Appl. Genet. 93, 975-981. doi: 10.1007/BF00224101

Crosatti, C., Polverino de Laureto, P., Bassi, R., and Cattivelli, L. (1999). The interaction between cold and light controls the expression of the cold-regulated barley gene cor $14 \mathrm{~b}$ and the accumulation of the corresponding protein. Plant Physiol. 119, 671-680. doi: 10.1104/pp.119.2.671

Dhillon, T., Pearce, S. P., Stockinger, E. J., Distelfeld, A., Li, C., Knox, A. K., et al. (2010). Regulation of freezing tolerance and flowering in temperate cereals: the VRN-1 connection. Plant Physiol. 153, 1846-1858. doi: 10.1104/pp.110.15 9079

Dhillon, T., and Stockinger, E. J. (2013). Cbf14 copy number variation in the A, B, and D genomes of diploid and polyploid wheat. Theor. Appl. Genet. 126, 2777-2789. doi: 10.1007/s00122-013-2171-0 
Dobrev, P. I., and Kamínek, M. (2002). Fast and efficient separation of cytokinins from auxin and abscisic acid and their purification using mixed-mode solidphase extraction. J. Chromatogr. A 950, 21-29. doi: 10.1016/S0021-9673(02) 00024-9

Dobrev, P. I., and Vankova, R. (2012). "Quantification of abscisic acid, cytokinin, and auxin content in salt-stressed plant tissues," in Plant Salt Tolerance: Methods and Protocols, Methods in Molecular Biology, eds S. Shabala and T. A. Cuin (New York City, NY: Humana Press), 251-261. doi: 10.1007/978-1-61779986-0

Du, X., Zhao, X., Li, X., Guo, C., Lu, W., Gu, J., et al. (2013). Overexpression of TaSRK2C1, a wheat SNF1-related protein kinase 2 gene, increases tolerance to dehydration, salt, and low temperature in transgenic tobacco. Plant Mol. Biol. Rep. 31, 810-821. doi: 10.1007/s11105-012-0548-x

Dubcovsky, J., and Dvorak, J. (2007). Genome plasticity a key factor in the success of polyploid wheat under domestication. Science 316, 1862-1866. doi: 10.1126/ science. 1143986

Eagles, H. A., Cane, K., and Trevaskis, B. (2011). Veery wheats carry an allele of Vrn-A1 that has implications for freezing tolerance in winter wheats. Plant Breed. 130, 413-418. doi: 10.1111/j.1439-0523.2011.01856.x

Fricano, A., Rizza, F., Faccioli, P., Pagani, D., Pavan, P., Stella, A., et al. (2009). Genetic variants of $\mathrm{HvCbf} 14$ are statistically associated with frost tolerance in a European germplasm collection of Hordeum vulgare. Theor. Appl. Genet. 119, 1335-1348. doi: 10.1007/s00122-009-1138-7

Galiba, G., Vanková, R., Tari, I., Bánfalvi, Z., Poór, P., Dobrev, P., et al. (2013). "Hormones, NO, antioxidants and metabolites as key players in plant cold acclimation," in Plant and Microbe Adaptations to Cold in a Changing World, eds R. Imai, M. Yoshida, and N. Matsumoto (New York, NY: Springer), 73-87. doi: 10.1007/978-1-4614-8253-6_7

Gonzalez-Guzman, M., Pizzio, G. A., Antoni, R., Vera-Sirera, F., Merilo, E., Bassel, G. W., et al. (2012). Arabidopsis PYR/PYL/RCAR receptors play a major role in quantitative regulation of stomatal aperture and transcriptional response to abscisic acid. Plant Cell 24, 2483-2496. doi: 10.1105/tpc.112.098574

Gulyás, Z., Boldizsár, A., Novák, A., Szalai, G., Pál, M., Galiba, G., et al. (2014). Central role of the flowering repressor ZCCT2 in the redox control of freezing tolerance and the initial development of flower primordia in wheat. BMC Plant Biol. 14:91. doi: 10.1186/1471-2229-14-91

Ha, S., Vankova, R., Yamaguchi-Shinozaki, K., Shinozaki, K., and Tran, L. S. P. (2012). Cytokinins: metabolism and function in plant adaptation to environmental stresses. Trends Plant Sci. 17, 172-179. doi: 10.1016/j.tplants. 2011.12.005

Hoffman, L., DaCosta, M., Ebdon, J. S., and Watkins, E. (2010). Physiological changes during cold acclimation of perennial ryegrass accessions differing in freeze tolerance. Crop Sci. 50, 1037-1047. doi: 10.2135/cropsci2009.06. 0293

Holková, L., Prášil, I. T., Bradáčová, M., Vítamvás, P., and Chloupek, O. (2009). Screening for frost tolerance in wheat using the expression of dehydrine genes Wcs 120 and Wdhnl 3 at $17^{\circ}$ C. Plant Breed. 128, 420-422. doi: 10.1111/j.14390523.2008.01606.x

Jeon, J., Kim, N. Y., Kim, S., Kang, N. Y., Novák, O., Ku, S. J., et al. (2010). A subset of cytokinin two-component signaling system plays a role in cold temperature stress response in Arabidopsis. J. Biol. Chem. 285, 23371-23386. doi: 10.1074/jbc.M109.096644

Juhász, Z., Boldizsár, Á., Nagy, T., Kocsy, G., Marincs, F., Galiba, G., et al. (2015). Pleiotropic effect of chromosome 5A and the mvp mutation on the metabolite profile during cold acclimation and the vegetative/generative transition in wheat. BMC Plant Biol. 15:57. doi: 10.1186/s12870-014-0363-7

Kalapos, B., Dobrev, P., Nagy, T., Vítámvás, P., Györgyey, J., Kocsy, G., et al. (2016). Transcript and hormone analyses reveal the involvement of ABA-signalling, hormone crosstalk and genotype-specific biological processes in cold-shock response in wheat. Plant Sci. 253, 86-97. doi: 10.1016/j.plantsci.2016. 09.017

Kim, H., Lee, K., Hwang, H., Bhatnagar, N., Kim, D. Y., Yoon, I. S., et al. (2014). Overexpression of PYL5 in rice enhances drought tolerance, inhibits growth, and modulates gene expression. J. Exp. Bot. 65, 453-464. doi: 10.1093/jxb/ert397

Knox, A. K., Li, C., Vágújfalvi, A., Galiba, G., Stockinger, E. J., and Dubcovsky, J. (2008). Identification of candidate CBF genes for the frost tolerance locus Fr-A $\mathrm{m} 2$ in Triticum monococcum. Plant Mol. Biol. 67, 257-270. doi: 10.1007/s11103008-9316-6
Kocsy, G., Athmer, B., Perovic, D., Himmelbach, A., Szűcs, A., Vashegyi, I., et al. (2010). Regulation of gene expression by chromosome 5A during cold hardening in wheat. Mol. Genet. Genomics 283, 351-363. doi: 10.1007/s00438010-0520-0

Kosová, K., Prášil, I. T., Vítámvás, P., Dobrev, P., Motyka, V., Floková, K., et al. (2012). Complex phytohormone responses during the cold acclimation of two wheat cultivars differing in cold tolerance, winter Samanta and spring Sandra. J. Plant Physiol. 169, 567-576. doi: 10.1016/j.jplph.2011.12.013

Kosová, K., Vítámvás, P., Planchon, S., Renaut, J., Vanková, R., and Prášil, I. T. (2013). Proteome analysis of cold response in spring and winter wheat (Triticum aestivum) crowns reveals similarities in stress adaptation and differences in regulatory processes between the growth habits. J. Proteome Res. 12, 4830-4845. doi: $10.1021 / \mathrm{pr} 400600 \mathrm{~g}$

Laemmli, U. K. (1970). Cleavage of structural proteins during assembly of head of bacteriophage T4. Nature 227, 680-685. doi: 10.1038/227680a0

Liu, Q., Kasuga, M., Sakuma, Y., Abe, H., Miura, S., Yamaguchi-Shinozaki, K., et al. (1998). Two transcription factors, DREB1 and DREB2, with an EREBP/AP2 DNA binding domain separate two cellular signal transduction pathways in drought- and low-temperature-responsive gene expression, respectively, in Arabidopsis. Plant Cell 10, 1391-1406.

Mao, X., Zhang, H., Tian, S., Chang, X., and Jing, R. (2010). TaSnRK2.4, an SNF1-type serine/threonine protein kinase of wheat (Triticum aestivum L.), confers enhanced multistress tolerance in Arabidopsis. J. Exp. Bot. 61, 683-696. doi: $10.1093 / \mathrm{jxb} / \operatorname{erp} 331$

Miller, A. K., Galiba, G., and Dubcovsky, J. (2006). A cluster of 11 CBF transcription factors is located at the frost tolerance locus Fr-Am2 in Triticum monococcum. Mol. Genet. Genomics 275, 193-203. doi: 10.1007/s00438-005-0076-6

Paolacci, A. R., Tanzarella, O. A., Porceddu, E., and Ciaffi, M. (2009). Identification and validation of reference genes for quantitative RT-PCR normalization in wheat. BMC Mol. Biol. 10:11. doi: 10.1186/1471-2199-10-11

Park, C., Seo, Y., and Park, C. (2015). Heat shock proteins: a review of the molecular chaperones for plant immunity. Plant Pathol. J. 31, 323-333. doi: 10.5423/PPJ. RW.08.2015.0150

Park, S.-Y., Fung, P., Nishimura, N., Jensen, D. R., Fujii, H., Zhao, Y., et al. (2009). Abscisic acid inhibits type 2C protein phosphatases via the PYR/PYL family of START proteins. Science 324, 1068-1071. doi: 10.1126/science.1173041

Pearce, S., Zhu, J., Boldizsár, Á, Vágújfalvi, A., Burke, A., Garland-Campbell, K., et al. (2013). Large deletions in the CBF gene cluster at the Fr-B2 locus are associated with reduced frost tolerance in wheat. Theor. Appl. Genet. 126, 2683-2697. doi: 10.1007/s00122-013-2165-y

Pecchioni, N., Kosová, K., Vítámvás, P., Prášil, I. T., Milc, J. A., Francia, E., et al. (2014). "Genomics of low-temperature tolerance for an increased sustainability of wheat and barley production," in Genomics of Plant Genetic Resources, eds R. Tuberosa, A. Graner, and E. Frison (Dordrecht: Springer), 149-183. doi: 10.1007/978-94-007-7575-6_6

Perez-Llamas, C., and Lopez-Bigas, N. (2011). Gitools: analysis and visualisation of genomic data using interactive heat-maps. PLOS ONE 6:e19541. doi: 10.1371/ journal.pone.0019541

Ried, J. L., and Walker-Simmons, M. K. (1993). Group 3 late embryogenesis abundant proteins in desiccation-tolerant seedlings of wheat (Triticum aestivum L.). Plant Physiol. 102, 125-131. doi: 10.1104/pp.102.1.125

Roberts, D. W. A. (1986). Chromosomes in cadet and rescue wheats carrying loci for cold hardiness and vernalization response. Can. J. Genet. Cytol. 28, 991-997. doi: 10.1139/g86-137

Santiago, J., Rodrigues, A., Saez, A., Rubio, S., Antoni, R., Dupeux, F., et al. (2009). Modulation of drought resistance by the abscisic acid receptor PYL5 through inhibition of clade A PP2Cs. Plant J. 60, 575-588. doi: 10.1111/j.1365-313X. 2009.03981.x

Sarhan, F., Ouellet, F., and Vazquez-Tello, A. (1997). Minireview the wheat wcsl20 gene family. A useful model to understand the molecular genetics of freezing tolerance in cereals. Physiol. Plant. 101, 439-446. doi: 10.1111/j.1399-3054. 1997.tb01019.x

Sears, E. R. (1953). Nullisomic analysis in common wheat. Am. Nat. 87, 245-252. doi: $10.1086 / 281780$

Shen, Q., Chen, C. N., Brands, A., Pan, S. M., and David Ho, T. H. (2001). The stress- and abscisic acid-induced barley gene HVA22: developmental regulation and homologues in diverse organisms. Plant Mol. Biol. 45, 327-340. doi: 10.1023/A:1006460231978 
Shen, Q., Uknes, S. J., and Ho, T. D. (1993). Hormone response complex in a novel abscisic acid and cycloheximide-inducible barley gene. J. Biol. Chem. 268, 23652-23660.

Soltész, A., Smedley, M., Vashegyi, I., Galiba, G., Harwood, W., and Vágújfalvi, A. (2013). Transgenic barley lines prove the involvement of TaCBF14 and TaCBF15 in the cold acclimation process and in frost tolerance. J. Exp. Bot. 64, 1849-1862. doi: 10.1093/jxb/ert050

Stockinger, E. J., Gilmour, S. J., and Thomashow, M. F. (1997). Arabidopsis thaliana CBF1 encodes an AP2 domain-containing transcriptional activator that binds to the C-repeat/DRE, a cis-acting DNA regulatory element that stimulates transcription in response to low temperature and water deficit. Proc. Natl. Acad. Sci. U.S.A. 94, 1035-1040. doi: 10.1073/pnas.94.3.1035

Sutka, J. (1981). Genetic studies of frost resistance in wheat. Theor. Appl. Genet. 59, 145-152. doi: 10.1007/BF00264968

Sutka, J., Kovács, G., and Veisz, O. (1986). Substitution analysis of the frost resistance and winter hardiness of wheat under natural and artificial conditions. Cereal Res. Commun. 14, 49-53.

Sutton, F., Ding, X., and Kenefick, D. G. (1992). Group 3 LEA Gene HVA1 regulation by cold acclimation and deacclimation in two barley cultivars with varying freeze resistance. Plant Physiol. 99, 338-340. doi: 10.1104/pp.99.1.338

Tian, S., Mao, X., Zhang, H., Chen, S., Zhai, C., Yang, S., et al. (2013). Cloning and characterization of TaSnRK2.3, a novel SnRK2 gene in common wheat. J. Exp. Bot. 64, 2063-2080. doi: 10.1093/jxb/ert072

Tóth, B., Galiba, G., Fehér, E., Sutka, J., and Snape, J. W. (2003). Mapping genes affecting flowering time and frost resistance on chromosome $5 \mathrm{~B}$ of wheat. Theor. Appl. Genet. 107, 509-514. doi: 10.1007/s00122-003-1275-3

Vágújfalvi, A., Aprile, A., Miller, A., Dubcovsky, J., Delugu, G., Galiba, G., et al. (2005). The expression of several Cbf genes at the Fr-A2 locus is linked to frost resistance in wheat. Mol. Genet. Genomics 274, 506-514. doi: 10.1007/s00438005-0047-y

Vágújfalvi, A., Crosatti, C., Galiba, G., Dubcovsky, J., and Cattivelli, L. (2000). Two loci on wheat chromosome $5 \mathrm{~A}$ regulate the differential cold-dependent expression of the cor14b gene in frost-tolerant and frost-sensitive genotypes. Mol. Gen. Genet. 263, 194-200. doi: 10.1007/s004380051160

Vágújfalvi, A., Galiba, G., Cattivelli, L., and Dubcovsky, J. (2003). The coldregulated transcriptional activator $\mathrm{Cbf} 3$ is linked to the frost-tolerance locus Fr-A2 on wheat chromosome 5A. Mol. Genet. Genomics 269, 60-67. doi: 10.1007/s00438-003-0806-6

Vágújfalvi, A., Kerepesi, I., Galiba, G., Tischner, T., and Sutka, J. (1999). Frost hardiness depending on carbohydrate changes during cold acclimation in wheat. Plant Sci. 144, 85-92. doi: 10.1016/S0168-9452(99)00058-8

Vanková, R., Kosová, K., Dobrev, P., Vítámvás, P., Trávníčková, A., Cvikrová, M., et al. (2014). Dynamics of cold acclimation and complex phytohormone responses in Triticum monococcum lines G3116 and DV92 differing in vernalization and frost tolerance level. Environ. Exp. Bot. 101, 12-25. doi: 10.1016/j.envexpbot.2014.01.002

Vazquez-Tello, A., Ouellet, F., and Sarhan, F. (1998). Low temperature-stimulated phosphorylation regulates the binding of nuclear factors to the promoter of Wcs120, a cold-specific gene in wheat. Mol. Gen. Genet. 257, 157-166. doi: $10.1007 / \mathrm{s} 004380050635$

Veisz, O., and Sutka, J. (1989). The relationships of hardening period and the expression of frost resistance in chromosome substitution lines of wheat. Euphytica 43, 41-45. doi: 10.1007/BF00037894
Vítámvás, P., Kosová, K., Prášilová, P., and Prášil, I. T. (2010). Accumulation of WCS120 protein in wheat cultivars grown at $9^{\circ} \mathrm{C}$ or $17^{\circ} \mathrm{C}$ in relation to their winter survival. Plant Breed. 129, 611-616. doi: 10.1111/j.1439-0523.2010. 01783.x

Vítámvás, P., Saalbach, G., Prášil, T., Čapková, V., Opatrná, J., and Ahmed, J. (2007). WCS120 protein family and proteins soluble upon boiling in coldacclimated winter wheat. J. Plant Physiol. 164, 1197-1207. doi: 10.1016/j.jplph. 2006.06.011

Vogel, J. T., Zarka, D. G., Van Buskirk, H. A., Fowler, S. G., and Thomashow, M. F. (2005). Roles of the CBF2 and ZAT12 transcription factors in configuring the low temperature transcriptome of Arabidopsis. Plant J. 41, 195-211. doi: 10.1111/j.1365-313X.2004.02288.x

Wu, J., Zhang, Y., Yin, L., Qu, J., and Lu, J. (2014). Linkage of cold acclimation and disease resistance through plant-pathogen interaction pathway in Vitis amurensis grapevine. Funct. Integr. Genomics 14, 741-755. doi: 10.1007/s10142014-0392-1

Xian, L., Sun, P., Hu, S., Wu, J., and Liu, J. H. (2014). Molecular cloning and characterization of CrNCED1, a gene encoding 9-cis-epoxycarotenoid dioxygenase in Citrus reshni, with functions in tolerance to multiple abiotic stresses. Planta 239, 61-77. doi: 10.1007/s00425-013-1963-4

Xiong, L., Lee, H., Ishitani, M., and Zhu, J. K. (2002). Regulation of osmotic stressresponsive gene expression by the LOS6/ABA1 locus in Arabidopsis. J. Biol. Chem. 277, 8588-8596. doi: 10.1074/jbc.M109275200

Xu, D., Duan, X., Wang, B., Hong, B., Ho, T., and Wu, R. (1996). Expression of a late embryogenesis abundant protein gene, HVA1, from barley confers tolerance to water deficit and salt stress in transgenic rice. Plant Physiol. 110, 249-257. doi: 10.1104/pp.110.1.249

Zhang, H., Li, W., Mao, X., Jing, R., and Jia, H. (2016). Differential activation of the wheat SnRK2 family by abiotic stresses. Front. Plant Sci. 7:420. doi: $10.3389 /$ fpls.2016.00420

Zhang, Z., Wang, Y., Chang, L., Zhang, T., An, J., Liu, Y., et al. (2016). MsZEP, a novel zeaxanthin epoxidase gene from alfalfa (Medicago sativa), confers drought and salt tolerance in transgenic tobacco. Plant Cell Rep. 35, 439-453. doi: 10.1007/s00299-015-1895-5

Zhang, H., Mao, X., Jing, R., Chang, X., and Xie, H. (2011). Characterization of a common wheat (Triticum aestivum L.) TaSnRK2.7 gene involved in abiotic stress responses. J. Exp. Bot. 62, 975-988. doi: 10.1093/jxb/erq328

Zhang, H., Mao, X., Wang, C., and Jing, R. (2010). Overexpression of a common wheat gene Tasnrk2.8 enhances tolerance to drought, salt and low temperature in Arabidopsis. PLOS ONE 5:e16041. doi: 10.1371/journal.pone.001 6041

Conflict of Interest Statement: The authors declare that the research was conducted in the absence of any commercial or financial relationships that could be construed as a potential conflict of interest.

Copyright (C) 2017 Kalapos, Novák, Dobrev, Vitámvás, Marincs, Galiba and Vanková. This is an open-access article distributed under the terms of the Creative Commons Attribution License (CC BY). The use, distribution or reproduction in other forums is permitted, provided the original author(s) or licensor are credited and that the original publication in this journal is cited, in accordance with accepted academic practice. No use, distribution or reproduction is permitted which does not comply with these terms. 